\title{
Euclid Near Infrared Spectro Photometer instrument concept and first test results at the end of phase $B$
}

Thierry Maciaszek, Ctr. National d'Études Spatiales, and Observatoire Astronomique de Marseille-Provence (France);

Anne Ealet, Ctr. de Physique des Particules de Marseille (France);

Knud Jahnke, Max-Planck-Institut für Astronomie (Germany);

Eric Prieto, Observatoire Astronomique de Marseille-Provence (France);

Rémi Barbier, Institut de Physique Nucléaire de Lyon (France);

Yannick Mellier, Institut d'Astrophysique de Paris (France), and Commissariat à l'Énergie Atomique (France);

Anne Costille, Franck Ducret, Christophe Fabron, Jean-Luc Gimenez, Robert Grange, Laurent Martin, Christelle Rossin, Tony Pamplona, Pascal Vola, Observatoire Astronomique de Marseille-Provence (France);

Jean Claude Clémens, Ctr de Physique des Particules de Marseille (France); Gérard Smadja, Institut de Physique Nucléaire de Lyon (France);

Jérome Amiaux, Jean Christophe Barrière, Michel Berthe, Commissariat à l'Énergie Atomique (France);

Adriano De Rosa, Enrico Franceschi, Gianluca Morgante, Massimo Trifoglio, Luca Valenziano INAF - IASF Bologna (Italy);

Carlotta Bonoli, Favio Bortoletto, Maurizio D'Alessandro, INAF - Osservatorio Astronimico di Padova (Italy);

Leonardo Corcione, Sebastiano Ligori, INAF - Osservatorio Astronomico di Torino (Italy);

Bianca Garilli, Marco Riva, INAF - IASF Milano (Italy);

Frank Grupp, Carolin Vogel, Max-Planck-Institut für extraterrestrische Physik (Germany);

Felix Hormuth, Gregor Seidel, Stefanie Wachter, Max-Planck-Institut für Astronomie (Germany);

Jose Javier. Diaz, Instituto de Astrofisica de Canarias (Spain);

Ferran Grañena, Cristobal Padilla, Institut de Física d'Altes Energies (IFAE) (Spain);

Rafael Toledo, Universidad Politécnica de Cartagena (Spain);

Per B. Lilje, University of Oslo; Bjarte G. B. Solheim, PROTOTECH (Norway);

Corinne Toulouse-Aastrup (SSC), Michael Andersen, DTU (Denmark);

Warren Holmes, Ulf Israelsson, Michael Seiffert, Carissa Weber, Augustyn Waczynski, NASA (USA)

René J. Laureijs, Giuseppe Racca, Jean-Christophe Salvignol, Paolo Strada, European Space Agency/ESTEC

On behalf of the Euclid Consortium

\begin{abstract}
The Euclid mission objective is to understand why the expansion of the Universe is accelerating by mapping the geometry of the dark Universe by investigating the distance-redshift relationship and tracing the evolution of cosmic structures. The Euclid project is part of ESA's Cosmic Vision program with its launch planned for 2020.

The NISP (Near Infrared Spectro-Photometer) is one of the two Euclid instruments and is operating in the near-IR spectral region (0.9-2 $\mu \mathrm{m})$ as a photometer and spectrometer. The instrument is composed of:

- a cold (135K) optomechanical subsystem consisting of a SiC structure, an optical assembly (corrector and camera lens), a filter wheel mechanism, a grism wheel mechanism, a calibration unit and a thermal control system

- a detection subsystem based on a mosaic of 16 Teledyne HAWAII2RG cooled to 95K with their front-end readout electronic cooled to $140 \mathrm{~K}$, integrated on a mechanical focal plane structure made with Molybdenum and Aluminum. The detection subsystem is mounted on the optomechanical subsystem structure

- a warm electronic subsystem $(280 \mathrm{~K})$ composed of a data processing / detector control unit and of an instrument control unit that interfaces with the spacecraft via a 1553 bus for command and control and via Spacewire links for science data

This presentation describes the architecture of the instrument at the end of the phase B (Preliminary Design Review), the expected performance, the technological key challenges and preliminary test results obtained on a detection system demonstration model.
\end{abstract}

Keywords: Euclid, Spectroscopy, Photometry, Infrared, Instrument, Dark Energy, Dark Matter, Baryon Acoustic Oscillation

\section{INTRODUCTION}

Euclid is a wide-field space mission concept dedicated to the high-precision study of dark energy and dark matter. Euclid will carry out an imaging and spectroscopic wide survey of the entire extra-galactic sky (15000 deg2) along with a deep survey covering 10-100 deg ${ }^{2}$. To achieve these science objectives the current Euclid reference design consists of a wide field telescope to be placed in L2 orbit by a Soyuz launch with a 6 years mission lifetime. The payload consists of a $1.2 \mathrm{~m}$ diameter 3-mirror telescope with two channels: a VISible imaging channel (VIS) and a Near Infrared Spectrometer and Photometer channel (NISP). Both instruments observe simultaneously the same Field of View (FoV) on the sky and the system design is optimized for a sky survey in a step-and-stare tiling mode.

The NISP Instrument is the near-infrared Spectrometer and Photometer operating in the 0.9-2.0 micron range at a temperature lower than $140 \mathrm{~K}$, except for detectors, cooled to $\sim 95 \mathrm{~K}$ or below. The warm electronics will be located in the service module, at a temperature around $20^{\circ} \mathrm{C}$.

The NISP instrument has two main observing modes: the photometric mode, for the acquisition of images with broad band filters, and the spectroscopic mode, for the acquisition of slitless dispersed images on the detectors.

In the photometer mode the NISP instrument images the telescope light in the wavelength range from $920 \mathrm{~nm}$ to $2000 \mathrm{~nm}$ (Y, J, H bands). The spatial resolution is required to be $0.3 \mathrm{arcsec}$ per pixel. The FoV of the instrument is $0.55 \mathrm{deg}^{2}$ having a rectangular shape of $0.763 \mathrm{deg} \times 0.722 \mathrm{deg}$.

In the spectrometer mode the light of the observed target is dispersed by means of grisms covering the wavelength range of $1.1-2 \mu \mathrm{m}$. In order to provide a flat resolution over the specified wavelength range four grisms are mounted in a wheel. These four grisms yield three dispersion directions tilted against each other by $90^{\circ}$ in order to reduce confusion from overlapping (due to slitless observing mode). The field and waveband definitions used in the individual configurations for spectroscopy and photometry are: 
- Three photometric bands:

1. Y Band: $920-1146 \mathrm{~nm}$

2. J Band: $1146-1372 \mathrm{~nm}$

3. H Band: $1372-2000 \mathrm{~nm}$

- Four Slitless spectroscopic bands:

1. Red $0^{\circ}$ disp. : $1250-1850 \mathrm{~nm}$

2. Red $90^{\circ}$ disp. : $1250-1850 \mathrm{~nm}$

3. Red $180^{\circ}$ disp. : $1250-1850 \mathrm{~nm}$

4. Blue $0^{\circ}$ disp. : $920-1250 \mathrm{~nm}$

The spectral resolution shall be higher than 250 for a 1 arcsec homogenous illumination object size. For such an object, the flux limit in spectroscopy shall be lower than $2 \times 10^{-16}$ erg. $\mathrm{cm}^{-2} . \mathrm{s}^{-1}$ at $1.6 \mu \mathrm{m}$ wavelength. As with all slitless spectrographs, the real resolution varies with the object size (the smaller the size is, higher the resolution is).

The image quality of the instrument in flight shall deliver a $50 \%$ radius encircled energy better than 0.3 " and a $80 \%$ one better than 0.7 ". There is a variation due to diffraction with wavelength.

The NISP budgets are presently the following:

The instrument sits in a box of $1 \times 0.6 \times 0.5 \mathrm{~m}$

The total mass of the instrument is $155 \mathrm{Kg}$

The instrument will produce $240 \mathrm{GBit}$ of data per day

\section{ARCHITECTURE}

\subsection{Functional Architecture}

The NISP functional architecture is given on Figure 2-1

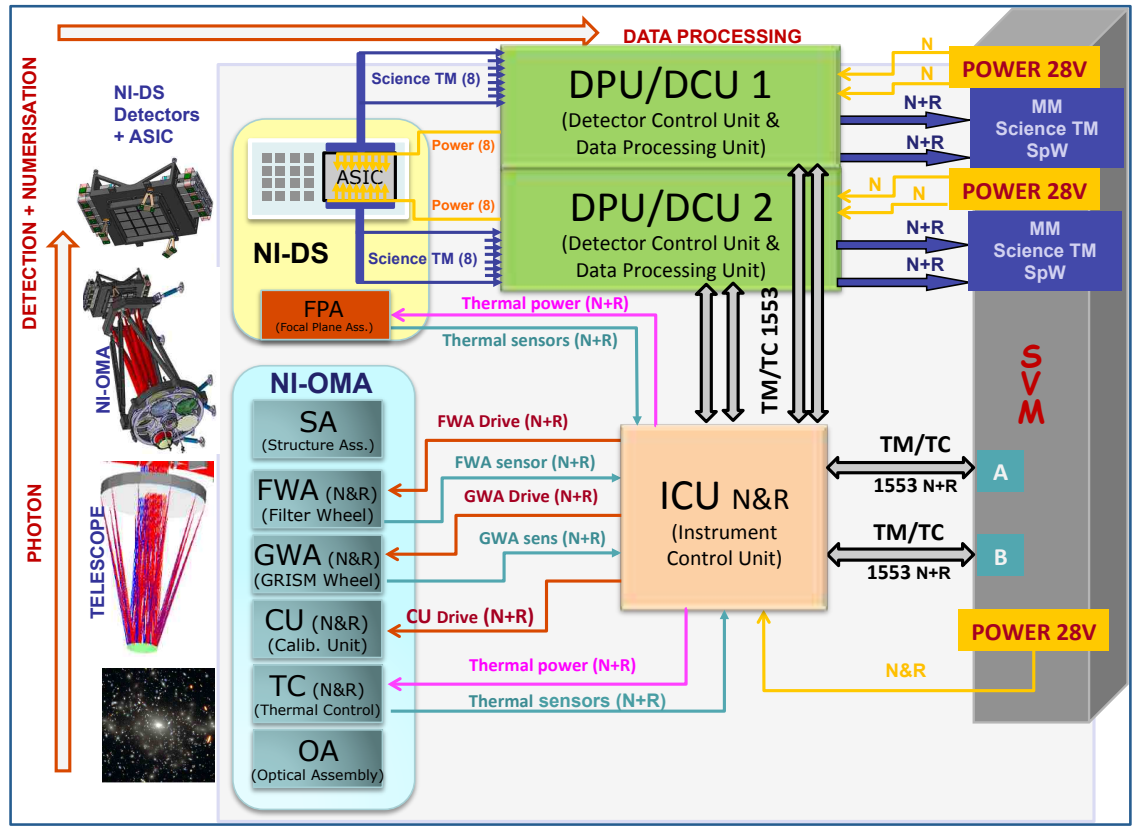

Figure 2-1: NISP functional scheme 


\subsection{Product Tree}

The NISP instrument consists of 3 main Assemblies:

- $\quad$ The NI-OMA (Opto-Mechanical Assembly), composed of the Mechanical Support Structure (NI-SA) and its thermal control (NI-TC), the Optical elements (NI-OA), the Filter Wheel Assembly (NI-FWA), the Grism Wheel Assembly (NI-GWA), the Calibration Unit (NI-CU). The NI-OMA structure supports the Optical elements, the calibration unit, the Filter and Grism Wheel Units and the detection system. It provides the thermo-mechanical interface towards the Euclid PLM.

- The Focal Plane Array (NI-FPA) and the Sensor Chip System (NI-SCS) compose the Detector System Assembly (NI-DS). The NI-DS comprises the 16 H2RG detectors and associated 16 ASICS (Sidecars), passively cooled at operating temperature $(<100 \mathrm{~K}$ for the detectors; $140 \mathrm{~K}$ for the ASICS Sidecar). Thermal stabilization of the detector is "naturally" obtained thanks to the very good thermal stability provided by the Euclid PLM at the NISP interfaces

- The Warm Electronics Assembly (NI-WE), composed of the Instrument Data Processing Unit and Control Unit (NI-DPU/DCU), and the Instrument Control Unit (NI-ICU). The NI-ICU is managing the commanding and the control of the instrument. It is interfaced with the satellite via a 1553 bus. The NI-DPU/DCU controls the Sensor Chip System and basic image processing such as co-adding (DCU function) and the science onboard data processing, the compression and transfer of scientific data to the S/C Mass Memory using Spacewire links (DPU function). The NI-DPU/DCU functions are regrouped in a single mechanical box for controlling 8 detectors. There are two NIDPU/DCU boxes.

The NISP hardware tree is summarized in the following Figure 2-2.

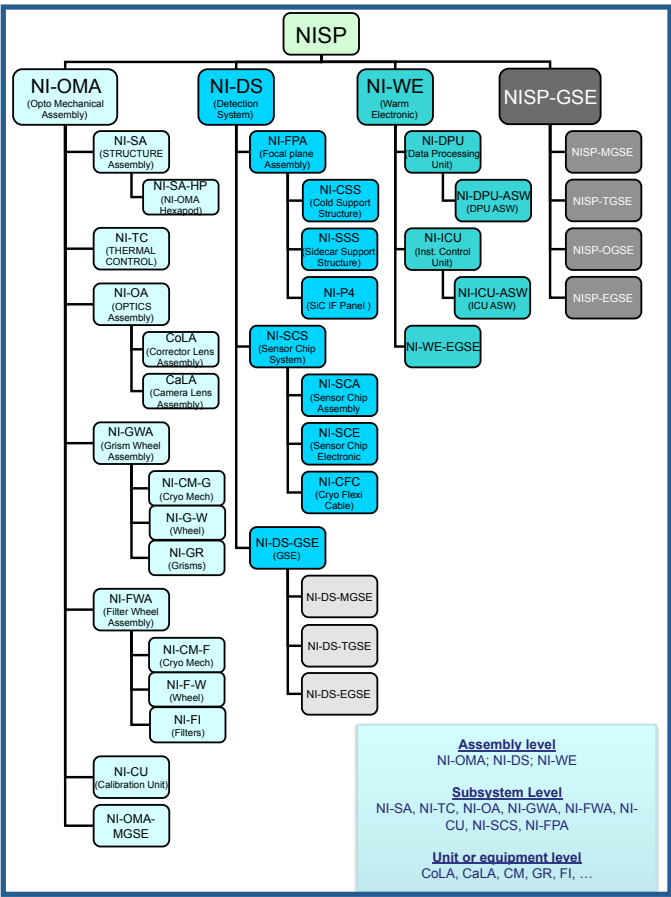

Figure 2-2: NISP Hardware Tree 


\section{OBSERVING SEQUENCE}

The instrument has a specific observing sequence that will be repeated throughout the mission lifetime.

The survey is decomposed into fields. To avoid confusion and increased spatial resolution each field is observed with 4-dithered frames for each band. Only one grism will be observed at each dither. The spectrum confusion is minimized thanks to the $90^{\circ}$ rotation of the dispersion within to same spectral band. During the nominal survey, only the 3 red grisms are used. The blue grism is used only during the deep survey.

Each sequence of exposure will consist of: $565 \mathrm{sec}$ for spectroscopy observation, respectively 121,116 and $81 \mathrm{sec}$ for the $\mathrm{Y}$, J, H photometric bands.

The wheels activate between each observation to set the instrument in the proper configuration. Due to the specificity of the H2RG detector, no shutter is needed to stop the integration. The shutter is included for dark calibration purposes (close position of the filter wheel) and to prevent accumulation of persistence during dithers and slews.

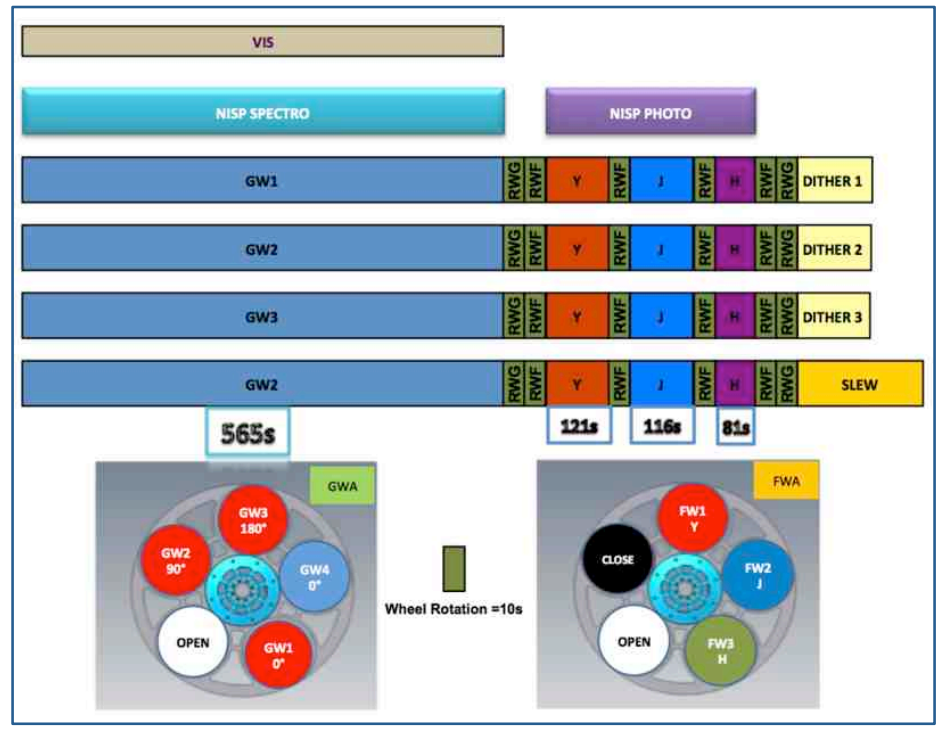

Figure 3-1: NISP Observing sequence 


\section{NISP OPTO-MECHANICAL ASSEMBLY (NI-OMA)}

This assembly has the function to filter, disperse and image the science telescope field of view. Figure 4-1 shows different views of the NI-OMA. The NI-SA (the structure) will be made of Silicon Carbide. The choice of the material is the result of a long trade-off between mainly carbon fiber, aluminum and SiC. The main driver was the very tight constraint of dimension stability of the system from AIV to the end of the mission. The system doesn't include any refocus mechanism independent of the VIS channel. Thus the stability of the co-focus of the two channels is a driver.

The first optical lens of the instrument (CoLA) is a corrector lens mounted on a SiC panel.

The filter wheel (NI-FWA) and the grism wheel (NI-GWA) are mounted inside a SiC box.

The 4 grisms are innovative very low dispersing elements of $13 \mathrm{ln} / \mathrm{mm}$ with aberration correction thanks to the curved groove pattern and to the variable line density.

The imaging optics (CaLA) is attached on the second side of the SiC box. This optical component is made of 3 lenses.

A 5 wavelengths calibration unit, with 5 LED's, shall permit to illuminate the focal plane directly. This will be utilized to calibrate the pixel-to-pixel flat field, the persistence, and the non-linearity of the detectors.

Operating temperature and thermal stability of the units are key drivers for the instrument. The structure (NI-SA) is by design thermally insulated from the PLM baseplate through Invar bipods and monopods. The total conductance from NI-OMA to the Baseplate is around $0.035 \mathrm{~W} / \mathrm{K}$. In such a way the heat exchanged conductively with the Baseplate should be minimized. In this configuration the units inside the NI-OMA, and especially the optical lenses, are less sensitive to fluctuations and can exploit the whole instrument thermal mass to operate in a more stable condition. Cooling at the required operating temperatures is provided mainly by heat extraction through two conductive thermal interfaces provided by the PLM located on the NI-DS (see below) and, partially, by radiative exchange with the PLM background. The structure entirely made of SiC, given the good thermal conductivity of this material, ensures good temperature uniformity and an efficient heat extraction towards the main conductive interfaces.

This NI-OMA is controlled at a temperature around $130 \mathrm{~K}$ with a stability better than $0.3 \mathrm{~K}$ for the full mission operation, from ground to EOL.

Radiative loads from the PLM cavity environment are efficiently shielded by an MLI shroud that surrounds the whole instrument (see Figure 4-1). The internal and external surfaces of this blanket are black to minimize straylight contamination.

On the following figures, photos of different elegant breadboards of different critical subsystems are shown.

The main challenges for the development of this system are:

- The very large cryogenic optics: the diameters of these lenses are in the range of $140-170 \mathrm{~mm}$ with fragile materials such as CaF2. It is the very first time that such lenses will fly in cryogenic environment. An intensive qualification work has been conducted to achieve TRL5 by the end of phase B1.

- The Grism technology: due to the low-resolution need, the gratings need to have very low line density with a very shallow angle. None of the traditional technics could be competitive for a 140mm grating diameter. A new method using photolithography technics has been developed and achieved TRL5 level.

- The very large and massive wheels: due to the optics dimensions, the wheels shall be very large. Their diameter goes up to $475 \mathrm{~mm}$ for a weight of $10 \mathrm{~kg}$. These characteristics lead to a very challenging concept for the cryo-mechanism.

- The stiff and very stable structure: we need to have a very stable structure from ground alignment to end of life. The SiC has been selected for this.
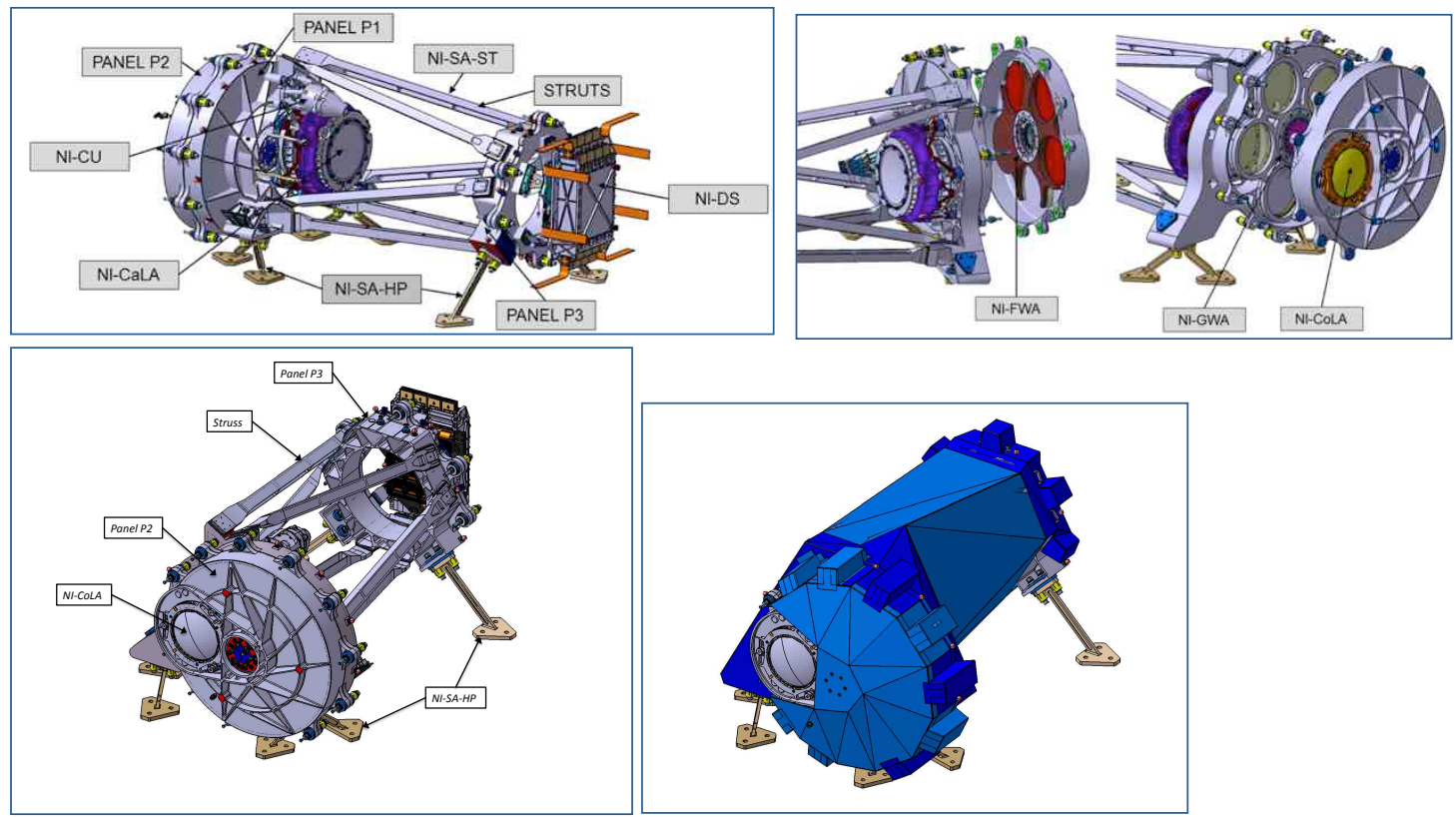

Figure 4-1: 4 views of the NI-OMA with the NI-DS mounted on it. 


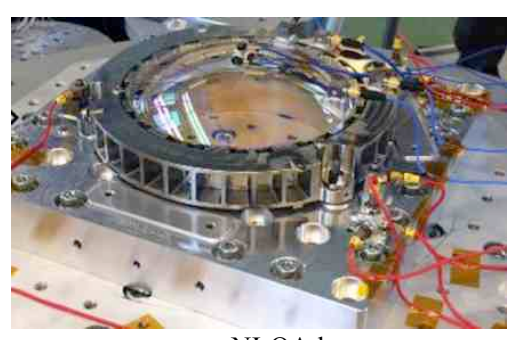

NI-OA lens

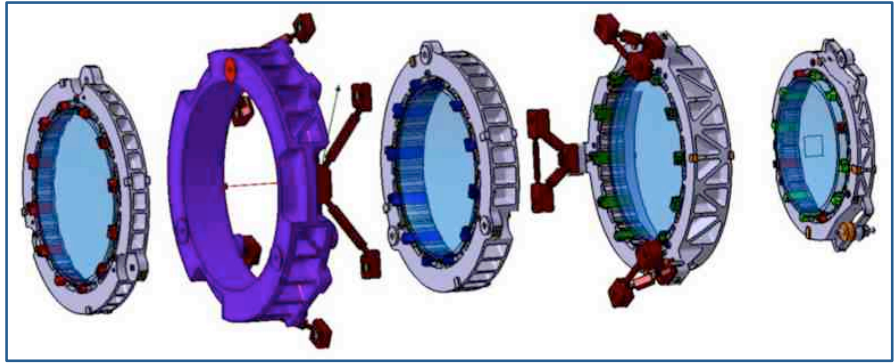

Exploded view of CaLA

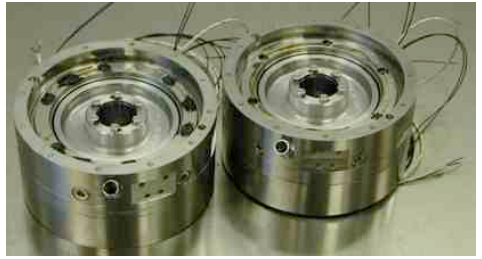

Cryomechanism

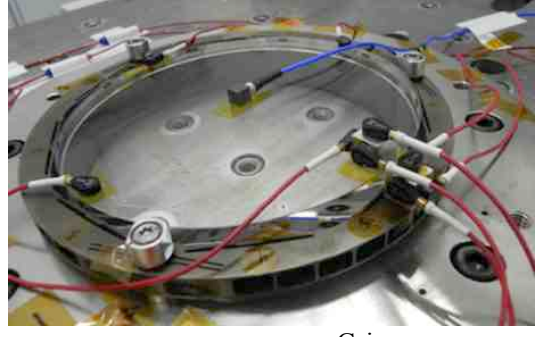

Grism

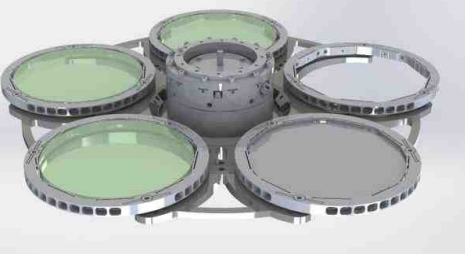

Filter wheel

Figure 4-2: Views of some NI-OMA subsystems. 


\section{THE NISP DETECTOR SYSTEM}

This assembly has the function to acquire the images by sampling the Field of View with an array of $4 \times 4$ IR sensors hybridized on multiplexers $(18 \mu \mathrm{m}$ pitch or 0.3 " on the sky) and read out by the Sidecar ASICs. It provides a light data processing to deliver digitalized data to the NI-WE. The Sensor chip System (SCE) composed of the H2RG sensor with $2.3 \mu \mathrm{m}$ cut-off, its $10 \mathrm{~cm}$ long cryo-flex cable and its ASIC is sequenced and read out by a Warm Electronic. From the photon conversion to digitalized data, each detection chain is independent.

All these SCE will be furnished by Teledyne under ESA and NASA contracts. The consortium is responsible to design the mosaic, integrate and qualify it.

The NI-DS is composed by (see the following figure):

1. The panel P4 made of $\mathrm{SiC}$ is the $\mathrm{I} / \mathrm{F}$ part of the NI-DS system and is screwed directly on the NI-OMA. The panel P4 is the support for the remaining NI-DS parts

2. The NI-CSS is the assembly of the focal plan plate, called Cold Plate, which supports the mosaic of $4 \times 4$ detectors. The Cold Plate is made of molybdenum and is held by 3 titanium blades. A baffle (for detector protection) also made of molybdenum is part of NI-CSS.

3. The NI-SSS is the support structure for the Sidecars (SCE). It is fixed onto the panel P4 by 3 blades made of invar. The NI-SSS is made of aluminum.

4. The NI-SCS composed of 4x4 SCAs (Sensor Chip Array), the CFCs (cryo-flex cable) and the SCEs (Sensor Chip Electronic which is the Sidecar ASIC).
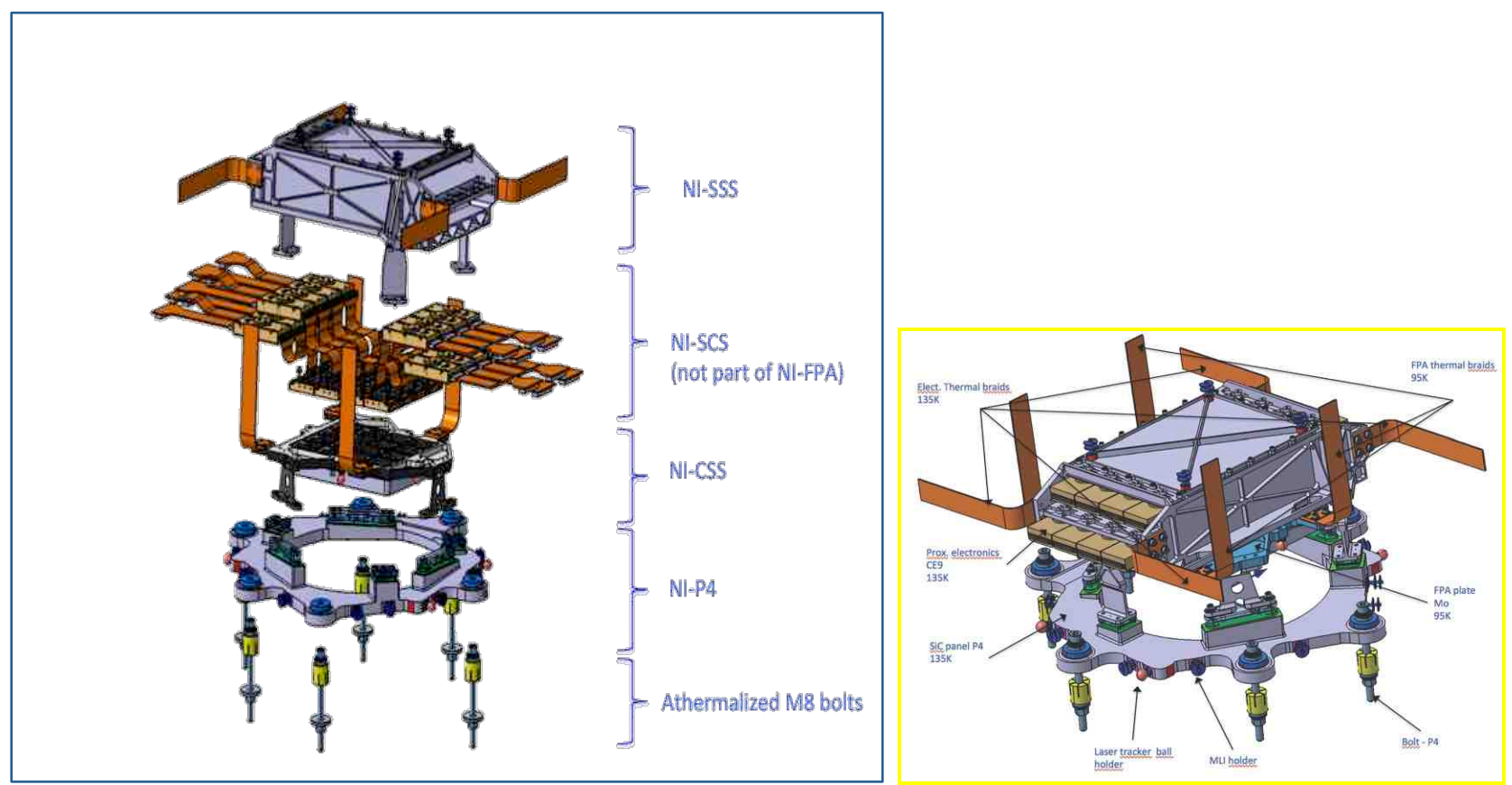

Figure 5-1: 4 views of the NI-DS

The operating temperature of the mosaic is lower than $100 \mathrm{~K}$ while each individual readout electronic (ASIC for digitization) operates at around $140 \mathrm{~K}$ Since the instrument units facing the detectors are controlled at a temperature below $135 \mathrm{~K}$, the resulting thermal emission up to $2.3 \mu \mathrm{m}$ ensures a very low thermal noise level. This configuration allows the optimization of the system thermal load on the satellite radiator and complies, at the same time, to all specification in term of noise.

The main challenges of this sub-system are:

- to integrate a mosaic of 16 detectors with a $0.3 \mathrm{~mm}$ range gap between each pedestal

- to provide a thermal architecture to insure adequate temperature stability and good thermal decoupling between electronics and detector

- $\quad$ crosstalk minimization: Extensive demonstration work will be done early in the project

A large amount of detector characterization will be conducted early in the project using individual detector chains to obtain precise characteristics for noise, dark current, conversion gain, non linearity of the pixel response, QE, Inter pixel capacitance crosstalk, full-well capacity and persistence (latency).

A demonstration model, with 4 detectors is presently integrated. TB/TV, cold conducted susceptibility, cold deformation measurement and vibration tests are planned before the end of 2014 . 

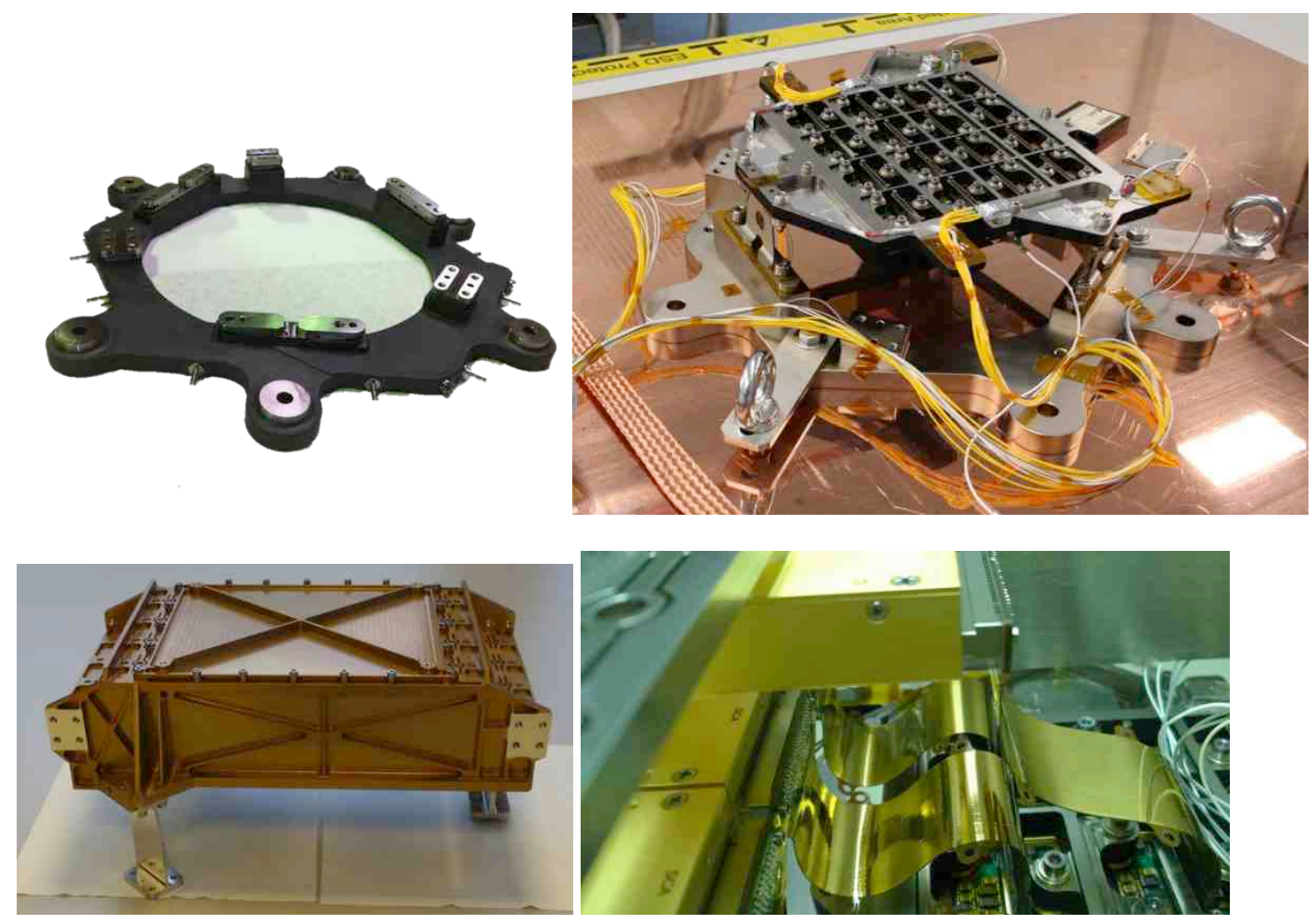

Figure 5-2: 4 views of the NI-DS demonstration model

The NISP focal plane presents several critical aspects, among them

- the operation of $16 \mathrm{x}$ independent devices to be synchronized avoiding readout phase jitter

- the presence of a long (about $8 \mathrm{~m}$ ) cable harness, partly at ambient temperature and partly in cryogenically ambient, interposed between each SCE/SCA pair and the driving electronics

- the need to cope with a global, restrictive, requirement on the final data quality

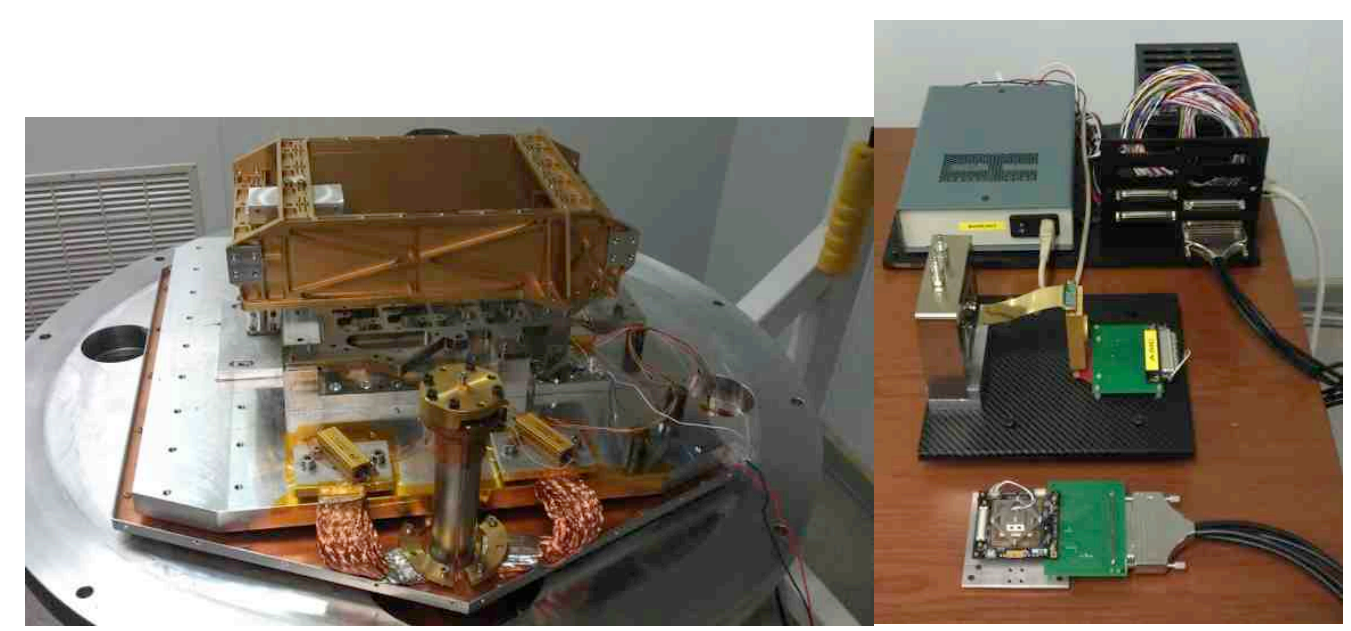

Figure 5-3: Left : electronics test-bench based on the Markury LTE multi-ASIC controller coupled to TIS cryo-ASIC and Euclid SCE. Right : Cryochamber holding the simulated NISP focal-plane system. The gold coated truss is the support for up to $16 \mathrm{x}$ SCEs at $135 \mathrm{~K}^{\circ}$, below the truss the molybdenum SCA cold plate at $100 \mathrm{~K}^{\circ}$. In front the cryo-cooler head.

SCA/SCE synchronism, at the level of a single master clock $(10 \mathrm{MHz})$ period, will be assured partly by SCE firmware specifically developed for the NISP application and partly by specific HW in the DCU electronics. Basically, all the SCE systems will be driven by a common master-clock and all writes to the SCE internal registers (configurations and command directives) will be synchronized by shift-registers started by a common pulse.

Data and power supply connection to each SCE is by an unusually long double-shielded cable harness. The length is primarily dictated by internal distribution inside the payload.

The kind of data communication (8x LVDS lines in parallel mode) and the requirements for the most critical power supply lines (VDDA and VDD2P5) have been already baselined and the harness is under study at Airbus DS. Several preliminary configurations (See Figure 5.3 above left side) have already been tested in order to evaluate critical aspects, such as master clock losses/duplication and critical power supply drops on the harness.

Further tests will be performed making use of flight representative cable harness and SCE power-supply systems as well as focal plane simulators and reference readout electronics. 
In particular the power-supply representative bread-board will mount the same DC-DC and continuous regulators, galvanic insulation system and ground/shielding concept as foreseen in flight. The SCA/SCE focal plane simulator (See Figure 5.3 above right side) will be based on 4x SCA bare multiplexers driven by $4 \mathrm{x}$ NISP SCE operated at flight foreseen temperatures in a cryo-chamber. SCE boot, configuration and data acquisition in the NISP standard mode (multi-accumulation) will be supported by a Markury LTE controller working in parallel with the power supply bread-board through $4 \mathrm{x}$ common cable harnesses.

The Markury LTE system has been already refurbished and tested by the contractor (Markury, US) to support the NISP specific SCE microcode and also tested (See left side of Figure 5.3 above) for proper operation with long cable harnesses and with the SCA mux/SCE operated at ambient temperature.

\section{THE NISP WARM ELECTRONIC}

The electronic is composed of::

- Two Data Processing Units (NI-DPU) each one is including:

- Detector Control Units that provide clock and power to the readout electronic. In addition, these units will preprocess the data using FPGA boards.

- Central Processor Unit that finalize the on board data processing, format the data and send them on to the SpaceWire Each DPU is hosting bus to the central spacecraft memory.

Data Processors based with 2 Maxwell SCS750 in cold redundancy

Data Routers

Data Buffers

8 DCU boards

Power Supply

Each DCU receives the data of one $2 \mathrm{kx} 2 \mathrm{k}$ detector from one SIDECAR and performs the low level pre-processing foreseen in HW consisting of

Pixel Coadding

Reference bias subtraction

Frame data buffering Coadded groups are then provided with 2 Spacewire (SpW) interfaces to 2 different Data Buffer boards for redundancy reason : in nominal conditions the DCU sends data to the Data Buffer board in the same section while in case of failure data can be sent to the Data Buffer in the other section. Each DCU can be controlled using a TMTC interface bus by each of the two processor boards : in nominal condition each processor is in charge of controlling all the DCUs in its section, so only one TMTC interface (X or Y) is active at the same time. The Data Buffer board receives data from $4+4 \mathrm{SPW}$ inputs and can be accessed by the processor on the cPCI bus for control and data retrieval. In nominal condition $4 \mathrm{SpW}$ only are active. The Data Buffer board allows the storage of a complete data set for spectroscopy and photometry. The Data Router board, interfaced with the processor on cPCI, implements

- Redundant SpW interface toward the MM with buffering

- Internal cPCI bus interface

- DCU TMTC Interfaces The architecture of power conditioning \& distribution system for NISP DPU/DCU has been defined to fulfill the following design constrains:

- Avoiding fault propagation

Optimizing DPU/DCU internal grounding scheme to provide the SIDECAR board with to very low noise power supply rails

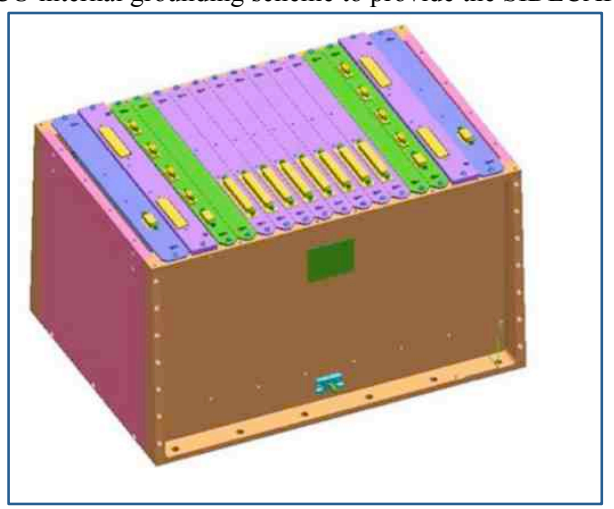

Figure 6-1: DPU

- One Instrument Control Unit (NI-ICU) in charge of:

- Interface with the spacecraft via a 1553 bus for the commanding of the NISP

- Housekeeping management

- General power supply

- Command signal to the cryo-mechanism, to the 5 LED's calibration source and to the NI-OMA and NI-DS heaters (heater constant power is applied in open loop with power setpoint determined by ground operators) 


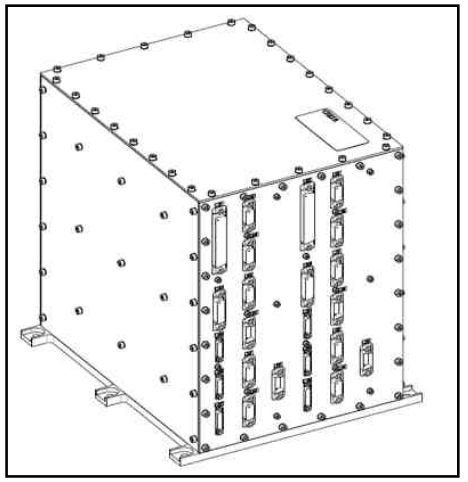

Figure 6-2: ICU

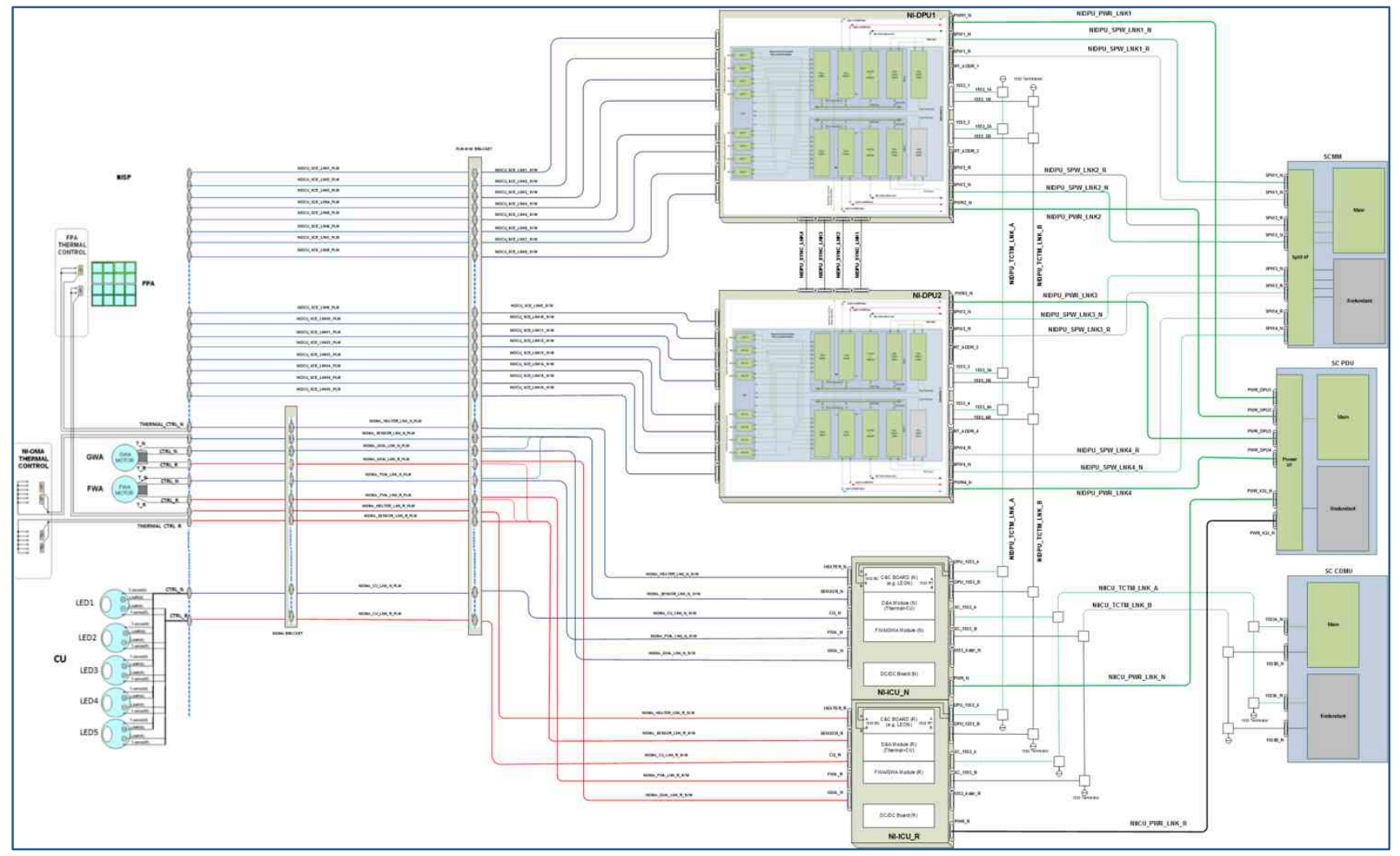

Figure 6-3: NISP electrical scheme

The warm electronic will be placed in the service module of the spacecraft at ambient temperature. A harness under Prime contractor responsibility provides the link with the NI-OMA and NI-DS. This cable will carry LVDS signal for scientific data, housekeeping signals, control command and power supply for equipment.

The main challenge of the warm electronics is to process the amount of data delivered by the detector during the integration of the following frame. The onboard data processing is complexified by the fact that the amount of downlink accepted to ground is very limited. Only final frames can be sent to ground, but as described later $\mathrm{HgCdTe}$ detectors deliver lots of frame to achieve final science performances.

The ICU Application SW (ASW) is devoted to manage the satellite/platform interface, the ICU/DPU interface and all the functionalities related to instrument commanding. It is in charge of the following functions:

- $\quad$ TM/TC exchange with S/C CDMU on Nom/Red 1553 link

- TC execution and distribution to NISP instrument units: NI-FWA electronics, NI-GWA electronics, NI-CMU electronics, NI-CU electronics, NI-TC electronics, NI-DPU, NI-DCU

- $\quad$ TM global instrument monitoring

- Manages time, TM time tagging and the high level instrument internal synchronizations

- Manages NISP operating modes

- Executes autonomous functions and FDIR algorithms and processes.

- Control of the calibration unit (ON/OFF, intensity level and current absorption handling)

- $\quad$ Control of Filter wheels (Reference position, position switch)

- Thermal control of the NI-FPA detector cold-plate through temperature sensors and heaters

- High level handling of macro-commands submission and termination verification to detector system

- Thermal control of the NI-OMA through temperature sensors and heaters 
- $\quad$ Manage the software maintenance, patch and dump functionalities

The ICU ASW is developed based on RTEMS real-time operative system, in the space-qualified version by EDISOFT.

Telecommand and Telemetry packets will be based on the Packet Utilization Services (PUS) standard, with the implementation of services tailored to the specific needs of the Euclid project.

A coordinated effort is in place with the Prime of the Spacecraft and with the VIS CDPU ASW team in order to ensure a common approach and, as far as possible, implementation of services between NISP and VIS, so that the SW interfaces with the Spacecraft can be simplified and standardized. The interface with the DPU is based on a MIL-STD-1553 bus, similar to the one used between the Spacecraft and the Euclid instruments. The SW interface and communication protocol is an internally defined one, with the aim of reducing as much as possible the load of management tasks on the DPU processor, since this resource is needed for the demanding data processing tasks.

\section{ONBOARD DATA PROCESSING}

The routine science NISP operations foresee a maximum of 20 cycles per day, each one composed by 4 dithers of $4 / 5$ exposures each, for a total maximum assigned science data telemetry of $240 \mathrm{Gbit} /$ day. This limited amount of allowed telemetry, together with the huge number of frames typically produced by IR detectors operated in multi-accumulation, have as a consequence the need to perform part of the processing pipeline directly on-board and to transfer to ground the final products for each exposure. Moreover final data must be also compressed to fit with the assigned telemetry throughput.

The data processing can be split into 2 main stages: stage 1 is implemented in the NI-DCU, directly interfaced to the SCS, where the first static basic pre-processing steps are performed, while stage 2, performed in NI-DPU, is devoted to the processing and compression of the final data frames. Figure 7.1 shows the Pre-processing HW structure connected to 1x SCS single pair (H2RG SCA + SCE) from a total of 16x located inside the SCS system.

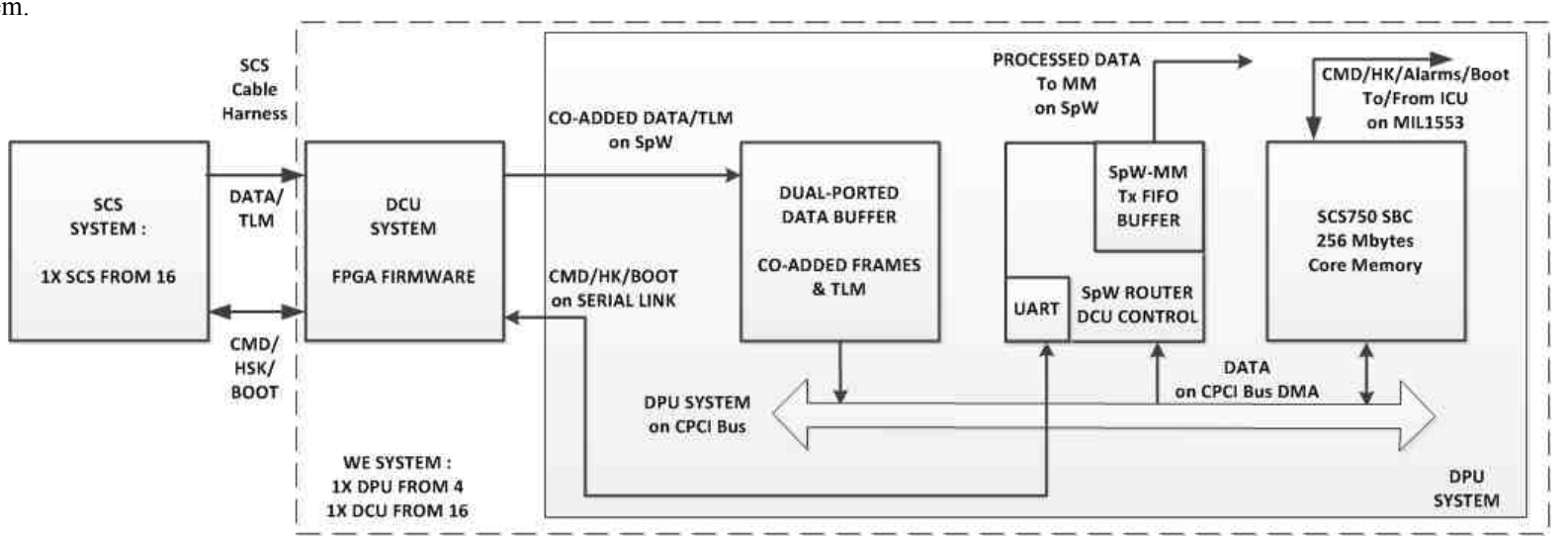

Figure 7-1: Pre-processing HW structure connected to 1x SCS single pair (H2RG SCA + SCE) from a total of 16x located inside the SCS system

As a result, the foreseen on-board pre-processing pipeline will be as depicted in the following figure, where the violet blocks represent the operations performed inside the DPUs. This operational flow is sequentially repeated to cover the 17 exposures (4 NISP-S +12 NISP-P +1 dark) to be performed during each single cycle.

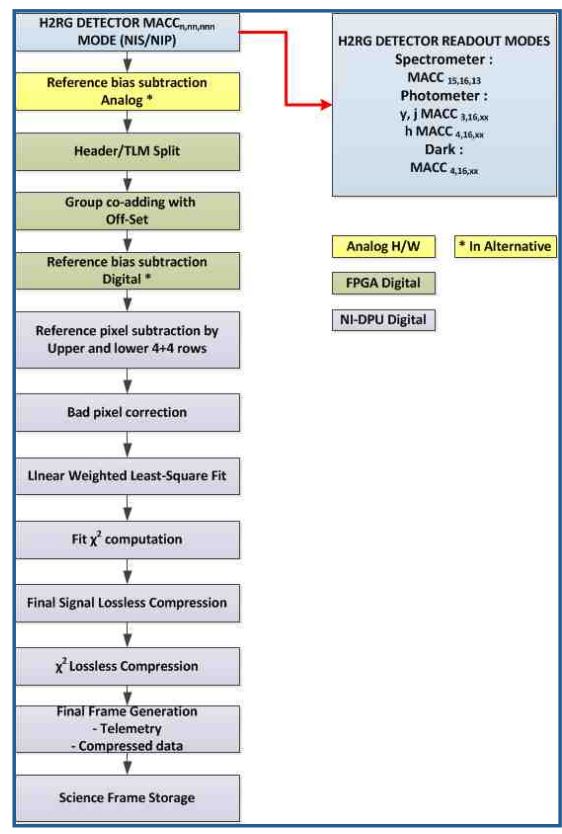

Figure 7-2: On-board data processing pipe-line for the Euclid NIS/NIP instrumental modes. The pipe-line is subdivided in three different sections on the base of the involved hardware, in the order: SCE analog hardware, FPGA hardware and sequential processing hardware 


\section{NISP PERFORMANCES}

The performances for both channels are derived mainly from the required science sensitivity:

To meet the science requirements, the imaging mode of the NISP instrument is required to have a depth of $\mathrm{Y}_{\mathrm{AB}}, \mathrm{J}_{\mathrm{AB}}$ and $\mathrm{H}_{\mathrm{AB}}=24$ mag $(5 \sigma)$ for a point source with a high image quality defined in terms of radii of encircled energy (EE50, EE80) of $(<0.30,<0.62) \operatorname{arcsec},(<0.30,<0.63)$ arcsec and $(<0.33$, $<0.70$ ) arcsec at the center of the $\mathrm{Y}, \mathrm{J}$ and $\mathrm{H}$ bands respectively.

To verify the compliance of the spectroscopic mode to science, a sensitivity on the detection of the Halpha line is computed to estimate the compliance with the requested limit flux of $2 \times 10^{-16} \mathrm{erg} \mathrm{cm}^{-2} \mathrm{~s}^{-1}$ at $3.5 \sigma$ of the emission line over the entire wavelength range.

Furthermore, to verify the impact of contamination of objects in the field, an advanced end-to-end simulation pipeline for spectroscopy is used with a realistic input galaxy distribution to evaluate the expected completeness and purity of the resulting selected spectroscopic sample.

The current radiometric budget has then to be built to verify these sensitivities with a best evaluation of the optical throughput information of each element and is given Figure 8-1:
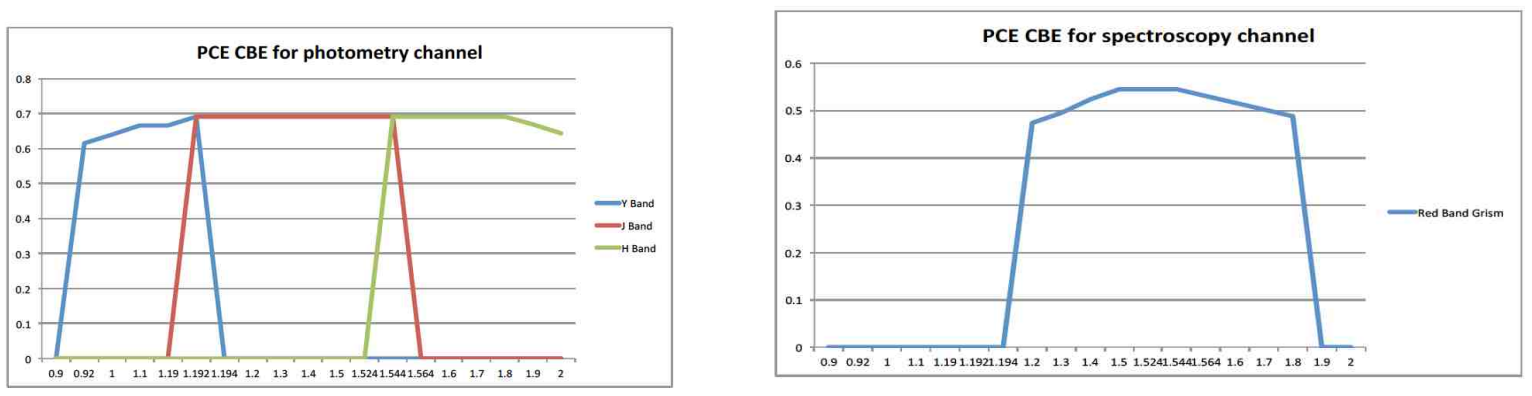

Figure 8-1: Best estimate of the PCE for photometry on the left and spectroscopy on the right

The background has been evaluated on the instrument from the derived budget from the sensitivity study. It is composed of the sky background and of the instrument background :

- $\quad$ the sky background, included contamination in spectroscopy

- $\quad$ the straylight coming from the scattered light on the optical and structural elements

- $\quad$ the ghosting coming from multiple reflections on the optical and structural elements

- $\quad$ the thermal background

- the total detector noise

Preliminary studies have been conducted and demonstrate the compliance at the system level. The main effects are coming from the CoLa reflection. The level of the CoLa ghost is at a relative intensity of $10^{-8}$ to central peak as illustrated in the following figure.

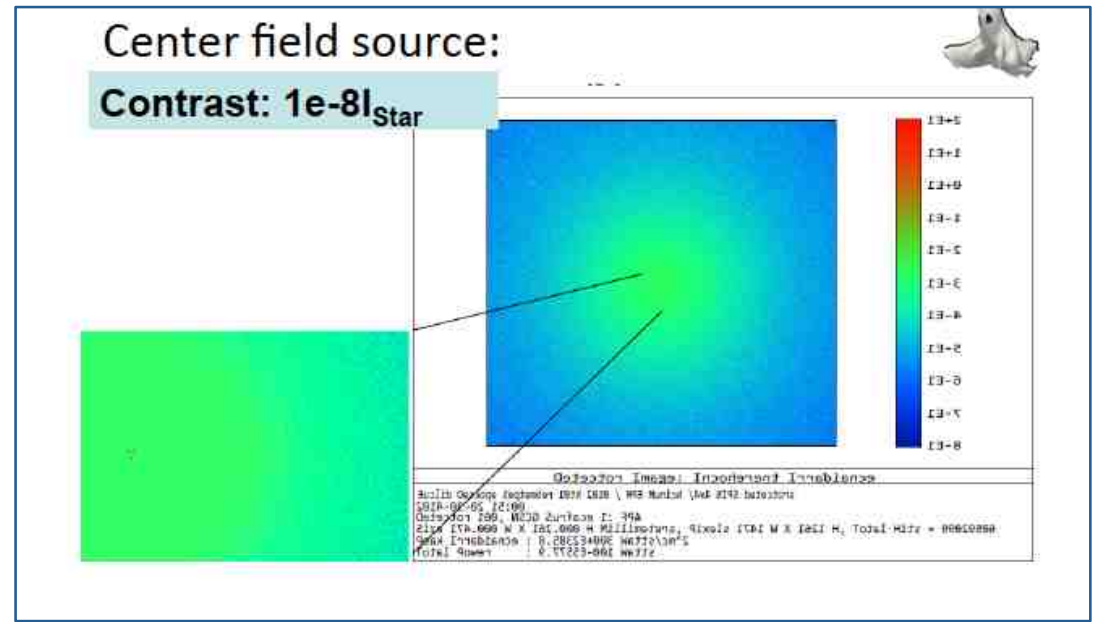

Figure 8-2: Ghost and Straylight from an object closed to the FOV in photometry. On the left, one can notice the ring above the PSF.

\section{The IR detector QE and noise performance}

The detector QE and the detector noise are a major concern for the future NISP performance. One important goal is to ensure not only mean specified values but also that $95 \%$ of pixels meet these requirements to ensure the efficiency of coverage in the full survey.

With some detectors already produced by Teledyne under ESA contract, some QE measurements done at NASA have shown excellent and flat QE within the $(0.92-2.3)$ band as seen on the following figure. 

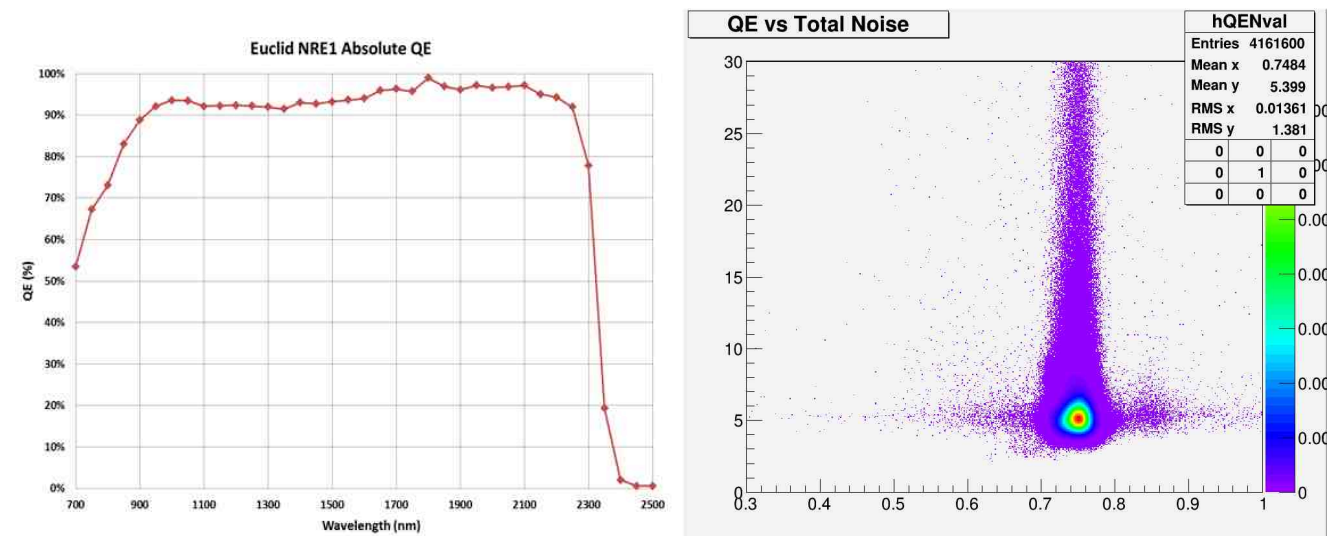

Figure 8-3: QE measurements on engineering detector between $920 \mathrm{~nm}$ and $2300 \mathrm{~nm}$ (left). Scatter plot of Total Noise in Fowler 16 and $100 \mathrm{~s}$ integration time and QE at 1.23 micron (right).

The mean dark has been shown to be very low around $0,005 \mathrm{e} / \mathrm{s}$ at $100 \mathrm{~K}$ with a sharp distribution and well inside NISP specifications. During the evaluation phase, 8 of the produced detectors demonstrated that SCA have passed the NISP requirements.

The parts show margins on QE and Total noise with a specification given on the 95\% percentile for the same pixels (QE .AND. noise)

The mean dark is very low and the distribution is sharp with $2.3 \mathrm{um}$ cut off SCA; measurement accuracy of $0.0045 \mathrm{e}-/ \mathrm{p} / \mathrm{s}$ is challenging (very long dark exposure with SCE stability).

\section{The Optical performance}

The optical design has been reviewed in term of required encircled energy with a full analysis.

The following figure shows evaluation compared to required values. It shows that NISP complies with its requirement.

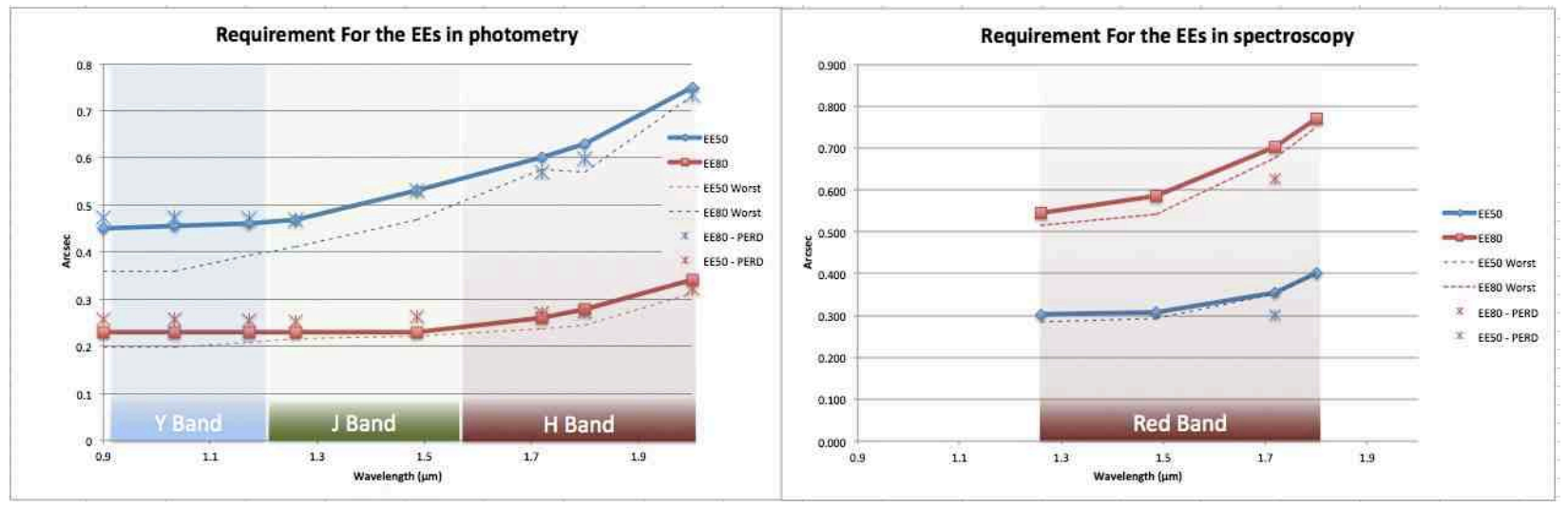

Figure 8-4: EE (Encircled Energy) performance evaluation 


\section{NISP ORGANISATION}

The following figure shows the organigram of the NISP. European Contributor countries are: France, Italy, Germany, Spain. Denmark and Norway, ESA for the engineering detectors and USA (NASA) for the flight detectors.

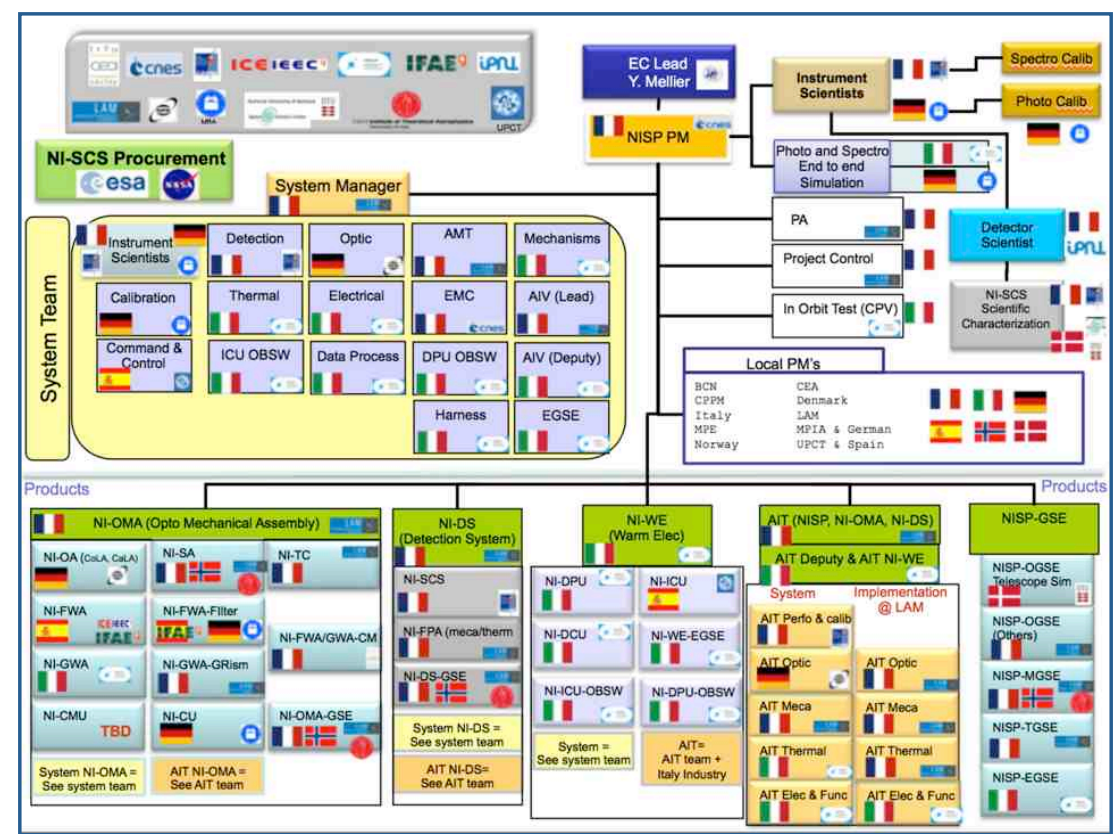

Figure 9-1: NISP Instrument organization

\section{NISP MODELS AND DEVELOPMENT}

The different models to be developed are the following :

A DM (Demonstration Model):

- $\quad$ to early demonstrate that the design of the NI-OMA \& NI-DS structure and thermal control behavior is correct and is as expected

- $\quad$ to do, as much as possible, all the environment test sequences expected for the FM at NI-DS and NIOMADA level

- to check, at NI-DS level, the EMC susceptibility of the NI-SCS (detector/flex cable/sidecars) and to prove the auto compatibility of

SCS working together and synchronized.

- to have a pre-validation of the different test procedures

- $\quad$ to train the AIT team

This model will be delivered to ESA as STM.

An Engineering and Qualification Model (EQM):

It will consist of one complete reduced instrument, including mechanisms, optics and related electronics. The EQM will be based on a four engineering

NI-SCS (detector+flexi+sidecar) completed by 12 STM NI-SCS. The purpose of this model is to fully qualify the design of the instrument.

- To allow subsystem and equipment functional, electrical, mechanical, thermal and performances qualification

- To validate the electrical Interfaces and the Command \& Control of the integrated instrument

- To proto-qualify the instrument with regard to the environment (qualification level with half duration)

- To prove the performances of the instrument with the telescope simulator.

This will be the first model with end to end performance measurements.

The NISP EQM is NOT a deliverable model, but at the end of the test campaign, the EQM it will be partially dismounted and reconfigured as an Avionic Model (AVM) to be delivered to ESA.

And at the end, THE FM model will be delivered to ESA.

\section{ACKNOWLEDGMENTS}

We thanks ALL the funding agencies of the NISP project: CNES, ASI, DLR, Spain agency, Norway agency, Denmark agency, ESA, NASA and ALL institutes which participates to this project.

\section{REFERENCES}

[1] R. Laureijs et al.,“ Euclid mission status”, SPIE Conference Series, [9143-16] (2014).

[2] E. Prieto et al., "Euclid near-infrared spectrophotometer instrument concept at the end of the phase A study", SPIE Conference Series, Vol. 8442, (2012). 